\title{
Extremal Values and Rankings of the Gutman Index of the Extended Double Stars
}

\author{
Mengmeng Feng ${ }^{1}$, Kelun Wang ${ }^{1}$ and Deqiang Wang ${ }^{1,2, *}$ \\ ${ }^{1}$ Department of Mathematics, Dalian Maritime University, Dalian 116026 \\ ${ }^{2}$ Nautical Science \& Technology Institute, Dalian Maritime University, Dalian 116026 \\ ${ }^{*}$ Corresponding author
}

\begin{abstract}
In 2016, Ivan Gutman et al. proposed the conception of the Gutman index of graphs. In this paper, based on the Gutman index of graphs, the vertex Gutman index of graphs is defined. On this basis, we obtained the maximum values and rankings of the vertex Gutman index of extended double stars, the extremal values and rankings of the Gutman index of the family of the extended double stars with a fixed order, and characterized the corresponding extremal vertices and extremal graphs.
\end{abstract}

Keywords-Gutman index; extended double stars; extremal value; ranking; extremal graph

\section{INTRODUCTION}

In graph theory, some topological indexes based on vertex degree-and-distance are well correlated with a variety of physico-chemical properties of organic compounds. In 1947, the American chemist Harold Wiener proposed the Wiener index based on the distance between vertices in order to estimate the boiling point of alkanes ${ }^{[1]}$. Since then, some researchers proposed many variants ${ }^{[2-6]}$ of Wiener index based on the vertex degree and the distance. In 1994, Gutman, Dobrynin and Kochetova introduced a weighted Wiener index of connected graphs, which was later called the Schultz index ${ }^{[7-}$ ${ }^{8]}$. In 1997, Klavzar and Gutman further defined the improved Schultz index by replacing the sum of degrees in Schultz index by the product of degrees ${ }^{[9]}$, which just was called the Gutman index ${ }^{[10]}$.

In this paper, our aim is to determine the extremal values and rankings of the vertex Gutman index of the extended double stars and the Gutman index of the family of the extended double stars with a fixed order, and characterize the corresponding extremal vertices and extremal graphs.

\section{DEFINITIONS AND NotATIONS}

Definition 1. ${ }^{[10]}$ Let $T=(V, E)$ be a graph, $d(u)$ and $d(u, v)$ denote the degree of the vertex $u$ and the distance between the vertices $u$ and $v$. $G(T)=\sum_{\{u, v\} \in V(T)}(d(u) \cdot d(v)) d(u, v)$ is called the Gutman index of the graph $T$.

Definition 2. Let $T=(V, E)$ be a graph. For any $u \in V$, the Gutman index of the vertex $u$ is defined as

$$
G(u)=d(u) \sum_{v \in V(T)} d(v) \cdot d(u, v)
$$

By Definition 1 and Definition 2, the Gutman index of the graph $T$ also can be expressed as

$$
\begin{aligned}
G(T) & =\frac{1}{2} \sum_{u \in V(T)}\left(d(u) \sum_{v \in V(T)} d(v) d(u, v)\right) \\
& =\frac{1}{2} \sum_{u \in V(T)} G(u) .
\end{aligned}
$$

Definition 3. Let $P_{r}=d_{1} d_{2} \cdots d_{r}(r \geq 2)$ be a path, ${ }^{u_{0}}$ and $v_{0}$ be the centers ofstar $S_{1, n_{1}}$ and $S_{1, n_{2}}$ respectively. The graph (denoted as $S_{n_{1}, n_{2}}^{(r)}$ ) composed by connecting $d_{1}$ to $u_{0}$ and $d_{r}$ to $v_{0}$ (see Figure 1) is called the extended double star, and the path $P_{r}$ is called the main path of $S_{n_{1}, n_{2}}^{(r)}$.

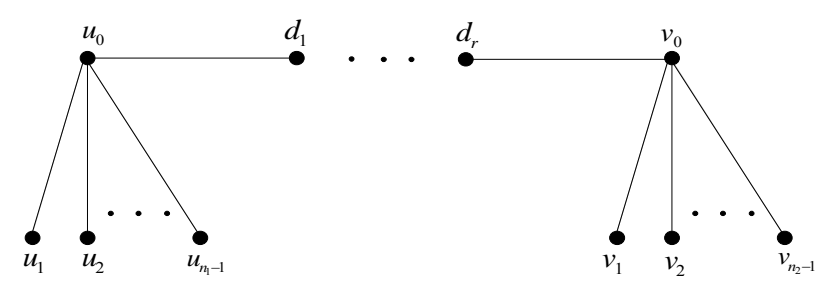

FIGURE I. THE EXTENDED DOUBLE STAR

As shown in Figure 1, the leaves of $S_{1, n_{1}-1}$ are denoted as $u_{1}, u_{2}, \cdots, u_{n_{1}-1}$, and the leaves of $S_{1, n_{2}-1}$ are denoted as $v_{1}, v_{2}, \cdots, v_{n_{2}-1}$.

Definition 4.Let $G\left(S_{n_{1}, n_{2}}^{(r)}, P_{r}\right)=\sum_{k=1}^{r} G\left(d_{k}\right)$.We refer to $G\left(S_{n_{1}, n_{2}}^{(r)}, P_{r}\right)$ as the contribution of the Gutman index of main path $P_{r}$ to extended double star $S_{n_{1}, n_{2}}^{(r)}$.

\section{MAIN RESULTS AND PROOFS}

\section{Theorem 1.}




$$
\begin{gathered}
\max \left\{G(u) \mid u \in V\left(S_{n_{1}, n_{2}}^{(r)}\right)\right\} \\
=\left\{\begin{array}{cc}
G\left(u_{0}\right)=G\left(v_{0}\right)=n_{1}^{2}+(2 r+3) n_{1} n_{2}-\left(r^{2}-3\right) n_{1}, & n_{1}=n_{2} ; \\
G\left(u_{0}\right)=n_{1}^{2}+(2 r+3) n_{1} n_{2}-\left(r^{2}-3\right) n_{1}, & n_{1}>n_{2} ; \\
G\left(v_{0}\right)=n_{2}^{2}+(2 r+3) n_{1} n_{2}-\left(r^{2}-3\right) n_{2}, & n_{1}<n_{2} .
\end{array}\right.
\end{gathered}
$$

Proof. By Definition 2, we have:

$$
\begin{aligned}
& G\left(u_{0}\right)=n_{1}^{2}+(2 r+3) n_{1} n_{2}+\left(r^{2}-3\right) n_{1} \\
& G\left(v_{0}\right)=n_{2}^{2}+(2 r+3) n_{1} n_{2}+\left(r^{2}-3\right) n_{2} \\
& G\left(u_{i}\right)=3 n_{1}+(2 r+5) n_{2}+r^{2}+2 r-7 ; \\
& G\left(v_{j}\right)=3 n_{2}+(2 r+5) n_{1}+r^{2}+2 r-7 .
\end{aligned}
$$

Meanwhile, we also can get the Gutman index of the vertex $d_{k} \in P_{r}(k \in Z, 1 \leq k \leq r)$ :

$$
\begin{aligned}
G\left(d_{k}\right)= & 2\left[2 k^{2}+2\left(n_{1}-n_{2}-r-1\right) k\right. \\
& \left.+n_{1}+(2 r+3) n_{2}+r^{2}-3\right] .
\end{aligned}
$$

It is easy to see that $G\left(d_{k}\right)$ takes the maximum value at $k=1$ or $k=r$, and

$$
\begin{aligned}
& G\left(d_{1}\right)=2\left[3 n_{1}+(2 r+1) n_{2}+r^{2}-2 r-3\right] \\
& G\left(d_{r}\right)=2\left[3 n_{2}+(2 r+1) n_{1}+r^{2}-2 r-3\right] .
\end{aligned}
$$

We fist note that:

$$
\begin{aligned}
& G\left(u_{0}\right)-G\left(u_{i}\right) \\
= & -(2 r+2) n_{1}^{2}+\left((2 r+3) n+r^{2}+2 r-1\right) n_{1}-r^{2}-2 r+7 \\
> & 0 .
\end{aligned}
$$

Therefore, $G\left(u_{0}\right)>G\left(u_{i}\right)\left(i=1,2 \cdots, n_{1}-1\right)$. Similarly, there is $G\left(v_{0}\right)>G\left(v_{j}\right)\left(j=1,2, \cdots, n_{2}-1\right)$.

Then, comparing the size of $G\left(u_{0}\right)$ and $G\left(d_{1}\right)$ :

$$
\begin{aligned}
G\left(u_{0}\right)-G\left(d_{1}\right)= & -2(r+1) n_{1}^{2}+\left[(2 r+3) n+r^{2}+4 r-7\right] n_{1} \\
& -(4 r+2) n-2 r^{2}+4 r+6 \\
> & 0
\end{aligned}
$$

Therefore, $G\left(u_{0}\right)>G\left(d_{1}\right)=G\left(d_{r}\right)$. Similarly, there is $G\left(v_{0}\right)>G\left(d_{k}\right)(1 \leq k \leq r)$.
Eventually, since $G\left(u_{0}\right)-G\left(v_{0}\right)=\left(n_{1}-n_{2}\right)\left(n_{1}+n_{2}+r^{2}-3\right)$, we can get:

(i) If $n_{1}=n_{2}, G\left(u_{0}\right)=G\left(v_{0}\right)$, the maximum value is $G\left(u_{0}\right)=G\left(v_{0}\right)$;

(ii) If $n_{1}>n_{2}, G\left(u_{0}\right)>G\left(v_{0}\right)$, the maximum value is $G\left(u_{0}\right)$;

(iii) If $n_{1}<n_{2}, G\left(u_{0}\right)<G\left(v_{0}\right)$, the maximum value is $G\left(v_{0}\right)$.

Theorem 1 shows that the maximum values of the vertex Gutman index of the extended double stars are taken at the vertex with maximal degrees.

Theorem 2. If $n_{1}=n_{2}\left(n_{1}, n_{2} \geq 3\right)$, the rankings of the vertex Gutman index of the extended double star $S_{n_{1}, n_{2}}^{(r)}$ can be shown that:

$$
\begin{aligned}
& G\left(u_{0}\right)=G\left(v_{0}\right)>G\left(d_{1}\right)>G\left(d_{2}\right)>\cdots \\
& >G\left(d_{\left\lfloor\frac{r+1}{2}\right\rfloor}\right)=G\left(d_{\left\lceil\frac{r+1}{2}\right\rceil}\right)>G\left(u_{i}\right)=G\left(v_{j}\right) ; \\
& G\left(u_{0}\right)=G\left(v_{0}\right)>G\left(d_{r}\right)>G\left(d_{r-1}\right)>\cdots \\
& >G\left(d_{\left\lceil\frac{r+1}{2}\right\rceil}\right)=G\left(d_{\left\lfloor\frac{r+1}{2}\right\rfloor}\right)>G\left(u_{i}\right)=G\left(v_{j}\right) .
\end{aligned}
$$

Proof. If $n_{1}=n_{2}\left(n_{1}, n_{2} \geq 3\right)$, in view of Theorem 1, there is $G\left(u_{0}\right)=G\left(v_{0}\right)$ and $G\left(u_{i}\right)=G\left(v_{j}\right)$. Let

$$
f(x)=2\left[2 x^{2}-2(r+1) x+(2 r+4) n_{1}+r^{2}-3\right] .
$$

Let $f^{\prime}(x)=0$, Then we get the stagnation point $x^{*}=(r+1) / 2$. Since $f^{\prime \prime}(x)=4>0$, so we get $f\left(x^{*}\right)=\min _{1 \leq x \leq r}\{f(x)\}$ and

$$
\begin{aligned}
& f(1)>f(2)>\cdots>f\left(\left\lfloor\frac{n+1}{2}\right\rfloor\right) \geq f\left(\frac{n+1}{2}\right) \\
& \leq f\left(\left[\frac{n+1}{2}\right]\right)<\cdots<f(r-1)<f(r) .
\end{aligned}
$$

Note that $f(i)=G\left(d_{i}\right)(i=1,2, \cdots, r)$, there is

$$
\begin{aligned}
& G\left(d_{1}\right)>G\left(d_{2}\right)>\cdots>G\left(d_{\left\lfloor\frac{n+1}{2}\right\rfloor}\right) \\
& =G\left(d_{\left\lceil\frac{n+1}{2}\right\rceil}\right)<\cdots<G\left(d_{r-1}\right)<G\left(d_{r}\right) .
\end{aligned}
$$

(1) From Theorem 1, we have: 


$$
\begin{aligned}
& G\left(u_{0}\right)-G\left(d_{1}\right) \\
= & \left(n_{1}-2\right) r^{2}+2\left(n_{1}^{2}-2 n_{1}+2\right) r+4 n_{1}^{2}-11 n_{1}+6>0,
\end{aligned}
$$

i.e.,

(2) Proving

$$
\begin{aligned}
& G\left(u_{0}\right)>G\left(d_{1}\right), G\left(v_{0}\right)>G\left(d_{r}\right) . \\
& G\left(d_{\left[\frac{r+1}{2}\right]}\right)=G\left(d_{\left[\frac{r+1}{2}\right]}\right)>G\left(u_{i}\right)=G\left(v_{j}\right) .
\end{aligned}
$$

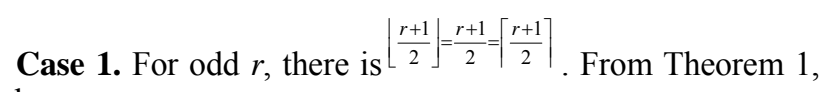
we have:

Case 2. For even $r$, there is $\left.\frac{r}{2}=\left[\frac{r+1}{2}\right] \frac{r+1}{2}<\frac{r+1}{2}\right] \frac{r+2}{2}$, and $G(r / 2)=G((r+2) / 2)$. From Theorem 1, we can get:

$$
\begin{aligned}
& G\left(d_{\frac{r}{2}}\right)-G\left(u_{i}\right)=2\left(n_{1}-2\right) r+1>0 . \\
& \text { Therefore, } G\left(d_{\left\lfloor\frac{r+1}{2}\right\rfloor}\right)=G\left(d_{\left\lceil\frac{r+1}{2}\right\rceil}\right)>G\left(u_{i}\right)=G\left(v_{j}\right) \text {. }
\end{aligned}
$$

Theorem 3. For $0<n_{2}-n_{1}<r-1$, the rankings of the vertex Gutman index of the extended double star $S_{n_{1}, n_{2}}^{(r)}$ can be shown that:

$$
\begin{aligned}
G\left(v_{0}\right) & >G\left(u_{0}\right)>G\left(d_{1}\right)>G\left(d_{2}\right)>\cdots>G\left(d_{n_{2}-n_{1}+1}\right) \\
& =G\left(d_{r}\right)>G\left(d_{n_{2}-n_{1}+2}\right)=G\left(d_{r-1}\right) \\
& >\cdots>G\left(d_{\left\lfloor\frac{r+1+n_{2}-n_{1}}{2}\right]}\right)=G\left(d_{\left[\frac{r+1+n_{2}-n_{1}}{2}\right]}\right)
\end{aligned}
$$$$
G\left(u_{i}\right)>G\left(v_{j}\right)\left(i=1,2, \cdots, n_{1} ; j=1,2, \cdots, n_{2}\right) .
$$

Proof. If $n_{1}<n_{2}$, from Theorem 1, there is $G\left(u_{0}\right)<G\left(v_{0}\right)$ and $G\left(u_{i}\right)>G\left(v_{j}\right)$. Let

$$
\begin{aligned}
& g(x)=2\left(2 x^{2}+2\left(n_{1}-n_{2}-r-1\right) x+n_{1}+(2 r+3) n_{2}+r^{2}-3\right) . \\
& \text { Let } g^{\prime}(x)=0 \text {. Then we get the stagnation point }
\end{aligned}
$$$$
y^{*}=\left(r+1+n_{2}-n_{1}\right) / 2(\in((r+1) / 2, r)) .
$$

$$
\begin{aligned}
\text { Since } & g^{\prime \prime}(x)=4>0, \text { so we get } \\
& g\left(y^{*}\right)=\min _{1 \leq x \leq r}\{f(x)\} \text { and } \\
& g(1)>g(2)>\cdots>g\left(\left|\frac{n+1}{2}\right|\right)>\cdots>g\left(\left\lfloor y^{*}\right\rfloor\right) \\
& =g\left(\left\lceil y^{*}\right\rceil\right)<\cdots<g(r-1)<g(r)
\end{aligned}
$$

Note that $f(i)=G\left(d_{i}\right)(i=1,2, \cdots, r)$, so

$$
\begin{aligned}
& G\left(d_{1}\right)>G\left(d_{2}\right)>\cdots>G\left(d_{\left[y^{*}\right\rfloor}\right) \\
& =G\left(d_{\left[y^{*} \mid\right.}\right)<\cdots<G\left(d_{r-1}\right)<G\left(d_{r}\right) .
\end{aligned}
$$

(1) From Theorem 1, we can get:

$$
\begin{aligned}
G\left(u_{0}\right)-G\left(d_{1}\right)= & n_{1} r^{2}+2 n_{1} n_{2} r+n_{1}^{2}+3 n_{1} n_{2}-3 n_{1} \\
& -2 r^{2}-4\left(n_{2}-1\right) r-6 n_{1}-2 n_{2}+6 \\
= & \left(n_{1}-2\right) r^{2}+2\left(n_{1} n_{2}-2 n_{2}+1\right) r \\
& +3 n_{1}\left(n_{2}-3\right)+n_{1}^{2}+2\left(r-n_{2}\right)+6 \\
> & 0,
\end{aligned}
$$

i.e., $G\left(u_{0}\right)>G\left(d_{1}\right)$.

(2) Since $r$ and $n_{2}-n_{1}+1$ are the symmetric points about the symmetric axis $x=\left(r+n_{2}-n_{1}+1\right) / 2$ of the quadratic function $f(x)$, so

$$
G\left(d_{m}\right)=f(m)=f\left(r+n_{2}-n_{1}-m\right)=G\left(d_{r+n_{2}-n_{1}-m}\right) .
$$

holds for any $m: n_{2}-n_{1}+1 \leq m \leq\left(r+n_{2}-n_{1}+1\right) / 2$.

Corollary 1. For $n_{2}-n_{1} \geq r-1$, the rankings of the vertex Gutman index of the extended double star $S_{n_{1}, n_{2}}^{(r)}$ are as follows:

$$
\begin{gathered}
G\left(v_{0}\right)>G\left(u_{0}\right)>G\left(d_{1}\right)>G\left(d_{2}\right)>\cdots>G\left(d_{r-1}\right)>G\left(d_{r}\right) ; \\
G\left(u_{i}\right)<G\left(v_{j}\right)\left(i=1,2, \cdots, n_{1} ; j=1,2, \cdots, n_{2}\right) .
\end{gathered}
$$

Corollary 2. For $n_{2}-n_{1}<0$, the rankings of the vertex Gutman index of the extended double star $S_{n_{1}, n_{2}}^{(r)}$ are as follows:

$$
\begin{gathered}
G\left(u_{0}\right)>G\left(v_{0}\right)>G\left(d_{r}\right)>G\left(d_{r-1}\right)>\cdots>G\left(d_{r+n_{2}-n_{1}}\right) \\
=G\left(d_{1}\right)>G\left(d_{r-1+n_{2}-n_{1}}\right)=G\left(d_{2}\right)>\cdots>G\left(d_{\left\lceil\frac{r+1+n_{2}-n_{1}}{2}\right\rceil}\right) ; \\
G\left(u_{i}\right)>G\left(v_{j}\right)\left(i=1,2, \cdots, n_{1} ; j=1,2, \cdots, n_{2}\right) .
\end{gathered}
$$


Theorem 4. The Gutman index of the extended double $\operatorname{star} S_{n_{1}, n_{2}}^{(r)}$ is:

$$
\begin{aligned}
G\left(S_{n_{1}, n_{2}}^{(r)}\right)= & 2 n_{1}^{2}+2 n_{2}^{2}+4(r+2) n_{1} n_{2}+\left(2 r^{2}+2 r-9\right) n_{1} \\
& +\left(2 r^{2}+2 r-9\right) n_{2}+\left(2 r^{3}-6 r^{2}-17 r+21\right) / 3
\end{aligned}
$$

Proof By Definition 4, the Contribution of the Gutman index of main path $P_{r}$ to extended double star $S_{n_{1}, n_{2}}^{(r)}$ is:

$$
\begin{aligned}
G\left(S_{n_{1}, n_{2}}^{(r)}, P_{r}\right)= & \sum_{k=1}^{r} G\left(d_{k}\right) \\
= & \sum_{k=1}^{r}\left[4 k^{2}+4\left(n_{1}-n_{2}-r-1\right) k\right] \\
& +\sum_{k=1}^{r}\left[2 n_{1}+(4 r+6) n_{2}+2 r^{2}-6\right] \\
= & \left(2 r^{2}+4 r\right)\left(n_{1}+n_{2}\right)+\left(4 r^{3}-6 r^{2}-22 r\right) / 3 .
\end{aligned}
$$

In the view of Definition 1 and Theorem 1, eventually, we can achieve:

$$
\begin{aligned}
G\left(S_{n_{1}, n_{2}}^{(r)}\right)= & {\left[G\left(u_{0}\right)+G\left(v_{0}\right)+\left(n_{1}-1\right) G\left(u_{i}\right)\right] / 2 } \\
& +\left[\left(n_{2}-1\right) G\left(v_{j}\right)+G\left(S_{n_{1}, n_{2}}^{(r)}, P_{r}\right)\right] / 2 \\
= & 2 n_{1}^{2}+2 n_{2}^{2}+4(r+2) n_{1} n_{2}+\left(2 r^{2}+2 r-9\right) n_{1} \\
& +\left(2 r^{2}+2 r-9\right) n_{2}+\left(2 r^{3}-6 r^{2}-17 r+21\right) / 3
\end{aligned}
$$

Let $n=n_{1}+n_{2}$, then the family of the extended double stars with a fixed order can be expressed as $\left\{S_{n_{1}, n-n_{1}}^{(r)}\right\}_{n_{1}=3}^{n-3}(n \geq 6, r \geq 2)$

Theorem 5. The extremal values of the Gutman index of the family of the extended double starswith a fixed order $n$ are:

$$
\begin{aligned}
& \max _{3 \leq n_{1} \leq n-3}\left\{G\left(S_{n_{1}, n-n_{1}}^{(r)}\right)\right\} \\
& =\left\{\begin{aligned}
G\left(S_{\frac{n-1}{2}, \frac{n+1}{2}}^{(r)}\right)= & G\left(S_{\frac{n+1}{2}, \frac{n-1}{2}}^{(r)}\right) \\
= & (r+3) n^{2}+\left(2 r^{2}+2 r-9\right) n \quad n \text { is odd; } \\
& +\left(2 r^{3}-6 r^{2}-20 r+18\right) / 3, \\
G\left(S_{\frac{n}{2}, \frac{n}{2}}^{(r)}\right)= & (r+3) n^{2}+\left(2 r^{2}+2 r-9\right) n \\
& +\left(2 r^{3}-6 r^{2}-17 r+21\right) / 3,
\end{aligned}\right. \\
& \min _{3 \leq n_{1} \leq n-3}\left\{G\left(S_{n_{1}, n-n_{1}}^{(r)}\right)\right\} \\
& =G\left(S_{3, n-3}^{(r)}\right)=G\left(S_{n-3,3}^{(r)}\right) \\
& =2 n^{2}+\left(2 r^{2}+14 r+3\right) n+\left(2 r^{3}-6 r^{2}-125 r-87\right) / 3 \text {. }
\end{aligned}
$$

Proof. By Theorem 4, we have:

$$
\begin{aligned}
G\left(S_{n_{1}, n-n_{1}}^{(r)}\right)= & -4(r+1) n_{1}^{2}+4(r+1) n n_{1}+2 n^{2} \\
& +\left(2 r^{2}+2 r-9\right) n+\left(2 r^{3}-6 r^{2}-17 r+21\right) / 3 .
\end{aligned}
$$

For $3 \leq x \leq n-3$, let

$$
\begin{aligned}
h(x)= & -4(r+1) x^{2}+4(r+1) n x+2 n^{2}+\left(2 r^{2}+2 r-9\right) n \\
& +\left(2 r^{3}-6 r^{2}-17 r+21\right) / 3 .
\end{aligned}
$$

By the property of the quadratic function, there are:

(1) $h(x)$ takes minimum value at $x=3$ or $x=n-3$. Because,

$$
\begin{aligned}
h(3) & =h(n-3) \\
& =2 n^{2}+\left(2 r^{2}+14 r+3\right) n+\left(2 r^{3}-6 r^{2}-125 r-87\right) / 3,
\end{aligned}
$$

so,

$$
\begin{aligned}
\min _{3 \leq n_{1} \leq n-3}\left\{G\left(S_{n_{1}, n-n_{1}}^{(r)}\right)\right\}= & \min _{x \in Z, 3 \leq x \leq n-3}\{h(x)\}=h(3)=h(n-3) \\
= & 2 n^{2}+\left(2 r^{2}+14 r+3\right) n \\
& +\left(2 r^{3}-6 r^{2}-125 r-87\right) / 3 .
\end{aligned}
$$

(2) If $n$ is odd, $h(x)$ takes maximum value at $x=(n-1) / 2$ or $x=(n+1) / 2$. So,

$$
\begin{aligned}
\max _{\substack{n \text { is odd } \\
3 \leq n_{1} \leq n-3}}\left\{G\left(S_{n_{1}, n-n_{1}}^{(r)}\right)\right\}= & \max _{x \in Z, 3 \leq n_{1} \leq n-3}\{h(x)\}=h\left(\frac{n-1}{2}\right)=h\left(\frac{n+1}{2}\right) \\
= & (r+3) n^{2}+\left(2 r^{2}+2 r-9\right) n \\
& +\left(2 r^{3}-6 r^{2}-20 r+18\right) / 3 .
\end{aligned}
$$

(3) If $n$ is even, $h(x)$ takes maximum value at $x=n / 2$. That is,

$$
\begin{aligned}
\max _{\substack{n \text { is even } \\
3 \leq n_{1} \leq n-3}}\left\{G\left(S_{n_{1}, n-n_{1}}^{(r)}\right)\right\}= & \max _{x \in Z, 3 \leq n_{1} \leq n-3}\{h(x)\}=h\left(\frac{n}{2}\right) \\
= & (r+3) n^{2}+\left(2 r^{2}+2 r-9\right) n \\
& +\left(2 r^{3}-6 r^{2}-17 r+21\right) / 3 .
\end{aligned}
$$

Theorem 5 shows that, in the family of the extended double starswith a fixed order $\mathrm{n}, \quad S_{\frac{n}{2}, \frac{n}{2}}^{(r)}$ is the extremal graphwith the maximum Gutman index for even $\mathrm{n}, S_{\frac{n-1}{2}, \frac{n+1}{2}}^{(r)}\left(\cong S_{\frac{n+1}{2}, \frac{n-1}{2}}^{(r)}\right)$ for odd $\mathrm{n}$; and $S_{3, n-3}^{(r)}\left(\cong S_{n-3,3}^{(r)}\right)$ isthe extremal graphwith the minimum Gutman index. 
Corollary 3. The rankings of the Gutman index of the family of the extended double stars with a fixed order $n$ are as follows:

(1) If $n$ is odd:

$$
\begin{aligned}
& G\left(S_{3, n-3}^{(r)}\right)<G\left(S_{4, n-4}^{(r)}\right)<\cdots<G\left(S_{\frac{n-1}{2}, \frac{n+1}{2}}^{(r)}\right) \\
& =G\left(S_{\frac{n+1}{2}, \frac{n-1}{2}}^{(r)}\right)>\cdots>G\left(S_{n-3,3}^{(r)}\right) .
\end{aligned}
$$

(2) If $n$ is even:

$$
G\left(S_{3, n-3}^{(r)}\right)<G\left(S_{4, n-4}^{(r)}\right)<\cdots<G\left(S_{\frac{n}{2}, \frac{n}{2}}^{(r)}\right)>\cdots>G\left(S_{n-3,3}^{(r)}\right) .
$$

Corollary 4. For $\left\{S_{n_{1}, n-n_{1}}^{(r)}\right\}_{n_{1}=3}^{n-3}(n \geq 6, r \geq 2)$, there is:

$2 n^{2}+\left(2 r^{2}+14 r+3\right) n+\left(2 r^{3}-6 r^{2}-125 r-87\right) / 3$

$\leq G\left(S_{n_{1}, n_{2}}^{(r)}\right)$

$\leq(r+3) n^{2}+\left(2 r^{2}+2 r-9\right) n+\left(2 r^{3}-6 r^{2}-17 r+21\right) / 3$.

(1) Left equality holds if and only if $S_{n_{1}, n_{2}}^{(r)} \cong S_{3, n-3}^{(r)} \cong S_{n-3,3}^{(r)}$;

(2) Right equality holds if and only if $S_{n_{1}, n_{2}}^{(r)} \cong S_{\frac{n}{2}, \frac{n}{2}}^{(r)}$.

Corollary 4 shows that the graph with maximum of the family of the extended double stars with a fixed order $n$ is $S_{\frac{n}{2}, \frac{n}{2}}^{(r)}$ and the graph with minimum is $S_{3, n-3}^{(r)}\left(S_{n-3,3}^{(r)}\right)$.

\section{CONCLUDING REMARKS}

In this paper, we discussed the vertex Gutman index for a typical kind of tree structure -- extended double stars. We obtained the extremal distribution of the vertex Gutman index of the extended double stars. For the family of the extended double stars with a fixed ordern,we got that $S_{\frac{n}{2}, \frac{n}{2}}^{(r)}$ is the extremal graphwith the maximum Gutman index for even $n$, $S_{\frac{n-1}{2}, \frac{n+1}{2}}^{(r)}\left(\cong S_{\frac{n+1}{2}, \frac{n-1}{2}}^{(r)}\right)$ for odd $n$; and $S_{3, n-3}^{(r)}\left(\cong S_{n-3,3}^{(r)}\right)$ isthe extremal graphwith the minimum Gutman index. These results revealed the relationship between the extremal values and the vertex degrees, which provided a new idea for the studying of the extremal values and extremal graphs of the Gutman index of other similar graphs.

\section{ACKNOWLEDGMENT}

This work was financially supported by National Natural Science Foundation of China (61472058) and the Fundamental Research Funds for the Central Universities (3132016322).

\section{REFERENCES}

[1] H. Wiener. Structural determination of paraffin boiling points. J. Am. Chem. Soc.pp. 17-20, 1947.

[2] P. Khadikar. The szeged index and analogy with the Wiener index. J. Chem. Inf. Comput. Sci.pp. 547-550, 1995。

[3] I. Gutman, N. Trinajstić. Graph theory and molecular orbitals total $\pi$ electron energy of alternant hydrocarnons. J. Chem. Phys. Lett. pp. 535538,1972 .

[4] I. Gutman, K. C. Das. The first Zagreb index 30 years after. J. MATCH Commum. Math. Comput. Chem. pp. 83-92, 2004.

[5] I. Gutman, O. Araujo, D. A. Morales. Bounds for the Randić connectivity index. J. Chem. Inf. Comput. Sci. pp. 572-579, 2000.

[6] G. Caporossi, I. Gutman, P. Hansen, L. Pavlović. Graphs with maximum connectivity index. J. Comput. Biol. Chem. pp. 85-90, 2003.

[7] A.A. Dobrynin, A.A. Kochetova. Degree distance of a graph: a degree analogue of the Wiener index. J. Chem. Inf. Comput. Sci. pp. 1082-1086, 1994.

[8] I. Gutman. Selected properties of the Schultz molecular topological index. J. Chem. Inf. Comput. Sci. pp. 1087-1089, 1994.

[9] S. Klavzar, I. Gutman. Wiener number of vertex-weighted graphs and a chemical application. J. Discrete Appl. Math. pp. 73-81, 1997.

[10] I. Gutman, B. Furtula, K. C. Das. On some degree-and-distance-based graph invariants of trees. J. Applied Mathematics and Computation. pp. $1-6,2016$. 